Bull. Mater. Sci, Vol. 6, No. i, September 1984, pp. 959-969. C) Printed in India.

\title{
Internal friction in hexagonal metalś
}

\author{
M K ASUNDI and C N RAO \\ Physical Metallurgy Division, Bhabha Atomic Research Centre, Trombay, Bombay 400085 , \\ India
}

\begin{abstract}
An overview of the studies of internal friction in hexagonal metals and alloys is presented. An outline of the experimental techniques of measurement and the atomistic mechanisms causing internal friction is also given.
\end{abstract}

Keywords. Anelasticity; internal friction; hexagonal metals.

\section{Introduction}

All materials possess the intrinsic ability to dissipate mechanical energy by some internal atomistic or microscopic processes. This property is called internal friction or damping capacity. Internal friction is mainly observed in systems undergoing periodic vibrations and its magnitude can be determined by the decay of free vibrations or of travelling waves in a test piece. In recent times, internal friction has become an important tool for investigating the atomistic processes in solids. One of the best known examples is the internal relaxation due to the diffusion of interstitial solutes in a bcc solid which can be conveniently studied by low cycle torsion pendulum measurements.

This paper gives a brief account of the various measures of internal friction, the experimental techniques of measurement and the mechanisms for internal friction followed by an overview of the studies on hexagonal materials. The results on zirconium and titanium alloys are given in greater detail due to the extensive studies made on these materials.

\section{Relaxation and internal friction}

A measure of the damping capacity is the quantity $\Delta W / W$ where $\Delta W$ is the energy loss per cycle and $W$ is the total vibrational energy. It is given by the integral $\int \sigma \mathrm{d} \varepsilon$ over one cycle where $\sigma$ is the applied stress and $\varepsilon$ is the resulting strain. For an ideal elastic body, there will be no dissipation of energy and the integral vanishes. When there is internal dissipation, stress and strain are no longer in phase with each other and the dissipation can be due to static or dynamic hysteresis (figure 1). For static hysteresis (figure la) part of the strain is irreversible and the internal friction will generally be independent of the frequency of vibrations, but will be strongly dependent on the amplitude of vibrations.

For dynamic hysteresis (figure 1b) relaxation occurs over a finite time by internal rearrangements in the material and depends on the rate of change of $\sigma$ and $\varepsilon$. Such a behaviour is called anelastic, a term introduced by Zener (1948). For small amplitude of 

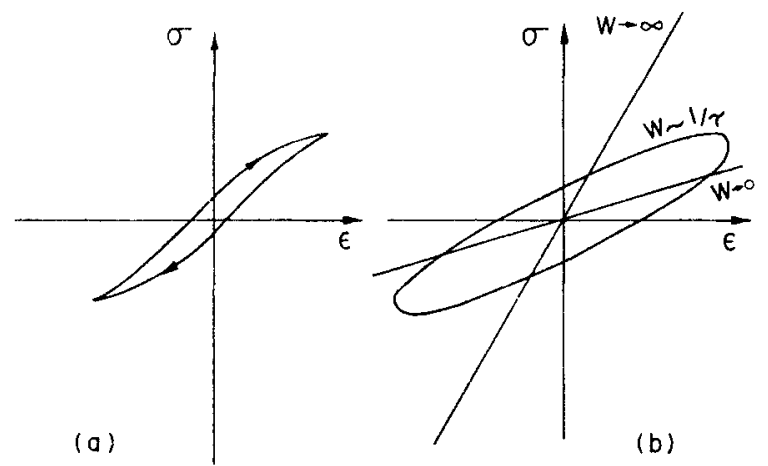

Figure 1. Schematic representation of mechanical hysteresis (a) static hysteresis (b) dynamic hysteresis.

the vibrations, the relation between $\sigma, \varepsilon$ and their time derivatives is linear and the damping will be independent of amplitude but strongly dependent on the frequency of vibration. At very high frequencies, there is little time for the relaxations to take place and no energy loss will be obtained. At very low frequencies, complete relaxation occurs and the behaviour of the system is purely elastic with a smaller relaxed value of the elastic modulus. At some intermediate frequency $\omega$ (of the order of $1 / \tau$ where $\tau$ is the relaxation time for the internal rearrangement) a maximum in internal friction will occur.

The various parameters used for quantitative specification of damping are dimensionless ratios which are independent of the size of the sample and the nature of the experimental system when the damping is independent of the amplitude of vibration.

\subsection{The logarithmic decrement $\delta$}

The damping of a system freely vibrating in a single mode can be expressed in terms of the rate of decay of the vibration amplitude. The logarithmic decrement is given by

$$
\delta=\frac{1}{n} \ln \left(A_{0} / A_{n}\right)
$$

where $A_{0}$ and $A_{n}$ are the amplitudes before and after $n$ cycles of vibration.

\section{$2.2 Q^{-1}$ value}

For a system undergoing forced vibrations, the amplitude magnification factor $Q$ at resonance and the relative width $\Delta \omega / \omega_{r}$ of the resonance (where $\omega_{r}$ is the resonance frequency) depend solely on the damping of the system. For the resonance width at half maximum they are related as

$$
Q^{-1}=\Delta \omega / \omega_{r}
$$

and provide a direct measure of the damping.

\subsection{Specific damping capacity, $P$}

If $W$ is the total energy of the oscillating system at the commencement of a cycle and $\Delta W$ the energy dissipated in the cycle, the specific damping capacity is defined as

$$
P=\Delta W / W \text {. }
$$




\subsection{Tangent of the phase angle}

In the case of linear damping, an applied sinusoidal force $f_{0} \sin \omega t$ produces a displacement $x_{0} \sin (\omega t-\phi)$, where $\phi$ is the phase lag between the applied force and displacement. The ratio of the components of strain out-of-phase and in-phase with the stress given by $\tan \phi$ can be used as a measure of the damping.

For small damping values, the different quantities defined above are approximately related to each other as

$$
\delta / \pi=Q^{-1}=P / 2 \pi=\tan \phi .
$$

\section{Experimental methods of measurement}

When considering a relaxation effect, four quantities are of interest; (i) the relaxation strength, which determines the peak height; (ii) the relaxation time which determines the peak position on the temperature scale; (iii) the shape of the peak which can be characterized in terms of the width at half maximum; and (iv) the modulus defect which causes a variation of the resonance frequency of the specimen in the temperature range of the damping peak.

Over the years, a variety of equipment have been built in various laboratories to measure internal friction. According to the method by which stresses are generated in the specimen, the techniques can be divided into resonance methods, pulse-echo methods and mechanical after-effect methods. Whereas the pulse-echo technique is restricted to high frequencies (over $1 \mathrm{MHz}$ ) and the after-effect method to very long relaxation times (over $10 \mathrm{sec}$ ), the resonance methods are useful in the range of low to medium frequencies $(0.01 \mathrm{~Hz}$ to $100 \mathrm{kHz})$. In a given type of experimental system one is usually restricted to a limited number of operating frequencies and hence to observe a relaxation process one has to vary the temperature. Thus, practically all the instruments described in the literature provide for measurements over a range of temperature.

A comprehensive description of the experimental methods can be found in the books by Nowick and Berry (1972) and De Batist (1972).

\section{Mechanisms of internal friction}

A number of atomistic and microscopic mechanisms can contribute to energy dissipation in solids. Some of these are: (a) stress-induced ordering of interstitial and substitutional atoms, commonly known as Snoek and Zener relaxations (Snoek 1941; Zener 1947), (b) grain boundary viscosity (Kê 1949), (c) thermoelastic damping (Waterman 1958), (d) dislocation damping (Read 1940), (e) magnetic damping in ferromagnetic materials (Cochardt 1959) and (f) electronic damping at low temperatures (Bommel 1954).

Covering the various aspects of relaxation processes and experimental results, extensive reviews have been published by Zener (1948), Nowick and Berry (1972), Nowick (1953), Entwistle (1962), De Batist (1972), Berry (1962), Mason (1966), Niblet and Wilks (1960), Wilks (1965), Burdett and Queen (1970), Fantozzi and Ritchie (1981) and Lucke and Granato (1957).

A brief account of the relaxations ascribed to point defects, dislocations and grain boundaries is given below: 


\subsection{Internal friction due to point defects}

Point defects produce anelastic behaviour through a process known as stress-induced ordering, in which the equilibrium configuration of a collection of defects in the crystal changes with time to a new state under applied stress. When the stress is removed, the change is reversed and with time the original state of order is restored. The occurrence of stress-induced ordering for point defects depends mainly on the ability of an applied sress to change the free energy associated with the presence of a defect in the crystal. If defects are present in several crystallographically equivalent positions whose free energies can be split by an external stress, a redistribution of the defects among these states will re-establish equilibrium. Only those defects which produce local strains having a lower symmetry than that of the lattice are capable of producing relaxation under shear. Such defects are called asymmetric defects or elastic dipoles.

An outstanding example of anelasticity produced by an elementary point defect acting as an elastic dipole is provided by the Snoek relaxation, first observed in $\alpha$-Fe containing small amounts of C or N (Snoek 1941, 1942). The Snoek effect has since been observed in a number of other bec transition metals containing impurity atoms (like $\mathrm{C}$, $\mathrm{N}, \mathrm{O}, \mathrm{H}$ ) in interstitial positions of the lattice. The Snoek peaks due to $\mathrm{O}, \mathrm{C}$ and $\mathrm{N}$ in niobium are shown in figure 2 (Hoffman and Wert 1966).

In $\alpha-\mathrm{Fe}$, the carbon atoms occupy the octahedral sites of the type $[1 / 2,0,0]$ (figure 3 ). The interstitial atom is an elastic dipole of $\langle 100\rangle$ tetragonal symmetry, which will cause stress induced ordering. The direction of the tetragonal dipole axis may lie along any one of the crystal cube axes $X, Y, Z$ depending on the particular site which the interstitial atom occupies. Sites which produce the tetragonal axis along the $X$ direction have been labelled $X$ in the figure (Similarly $Y$ and $Z$ sites). When an interstitial impurity makes thermally-activated jumps between neighbouring sites, which are $a / 2$ apart, the dipole axis re-orients by $90^{\circ}$ with each jump. Thus, the relaxation rate is related in a simple way to the mean jump rate of the interstitial atoms.
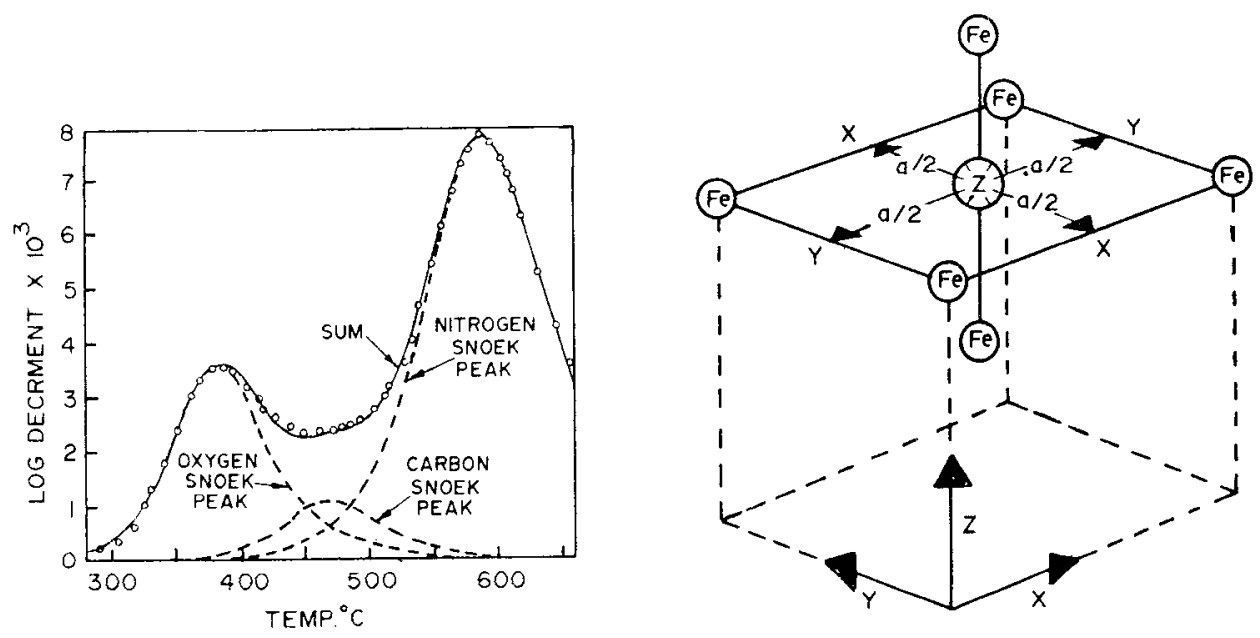

Figure 2. Snoek peaks in a $\langle 100\rangle$ crystal of niobium tested in longitudinal vibration at $80 \mathrm{kHz}$.

Figure 3. Solute atom (shaded) in an interstitial octahedral site of bcc Fe. 


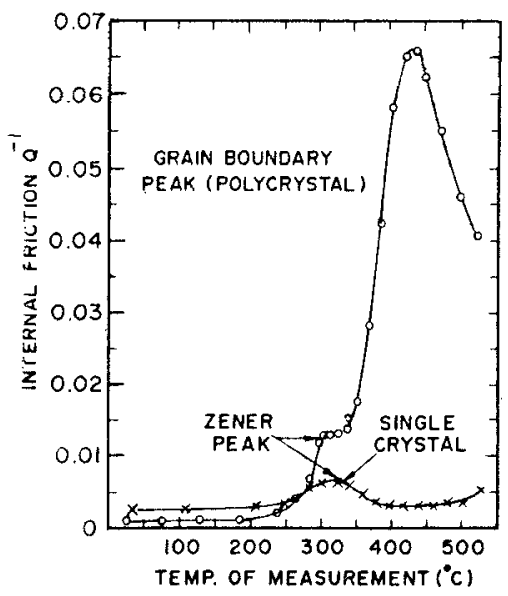

Figure 4. Zener peak in polycrystalline and single crystal specimens of $\alpha$-brass (torsional vibration at $0.5 \mathrm{~Hz}$ ).

Another important kind of point defect process, ascribed to stress-induced reorientation of solute pairs, is the Zener relaxation, first observed by Zener (1943) in a single crystal of $70-30 \alpha$-brass. Since then, similar relaxation peaks have been found in a number of binary (and other complex) alloys with fcc, bcc and hcp crystal structures. According to the pair reorientation theory of Zener (1947) asymmetric distortions can be expected around two adjacent solute atoms i.e. around a nearest neighbour pair. This enables splitting of free energies of differently oriented pairs by an applied stress. Figure 4 gives the Zener peak observed in $\alpha$-brass.

In addition to the solute-solute $(S-S)$ defect pair, interaction of substitutionalinterstitial $(S-I)$ pairs, and $I-I$ pairs may lead to internal friction peaks (see for example Nowick and Berry 1972).

\subsection{Relaxation involving dislocations}

A variety of relaxation peaks occur at temperatures, usually below room temperature, after plastic deformation. They are considered to be caused by the movement and interaction of dislocations with point defects.

In the vibrating-string model (Granato and Lucke 1956) the dislocation line is considered to behave like an elastic string fixed at two points in the lattice, restricted to move in two dimensions in the glide plane. A number of anelastic effects can be deduced from the model. One important result is that the anelastic properties of dislocations should be strongly frequency dependent. Changing the resonance frequency of dislocations by decreasing the free length of the vibrating string (introducing additional pinning centres by irradiation) affords an effective method of checking the results of the dislocation damping theory and to study the production and recovery of defects.

By considering the movement of the dislocation string in a periodic lattice potential - the Peierls potential-additional relaxation effects due to anelastic motion of segments of the dislocation line are predicted.

Dislocation motion described by means of kink diffusion (Brailsford 1961) along dislocation lines can also be used to explain dislocation damping caused by resonance effects as well as relaxation effects. 
The trapping of point defects in the stress field of a dislocation will have a profound effect on dislocation damping through variation in free dislocation length. A number of relaxation peaks observed in plastically deformed metals have been attributed to the interaction and motion of point defects and dislocations. The amplitude dependence of dislocation damping can be explained qualitatively in terms of dislocations anchored by point defects.

The Bordoni peak, first observed at low temperatures for deformed copper, has been studied in a number of metals. A second (Niblett-Wilks) peak associated with the Bordoni peak occurs at a lower temperature and is usually much smaller than the main peak (Niblett and Wilks 1956). A series of three peaks has been observed in deformed metals in the range between room temperature and the low temperature Bordoni peaks (Hasiguti et al 1962).

\subsection{Boundary relaxation processes}

Grain boundaries, domain boundaries and semi-coherent and incoherent interface boundaries between phases can result in relaxation effects giving an internal friction maximum when the boundary movement corresponds to the frequency of the applied stress field. Such effects will be observed at low frequencies and relatively high temperatures. The grain boundary peak in aluminium is shown in figure 5 .

\section{Internal friction studies in hexagonal metals}

\subsection{Relaxation effects in hep metals}

In the hexagonal close-packed structure, there are two possible locations for an interstitial (figure 6). The volume associated with the octahedral site is larger than that of the tetrahedral site and hence larger atoms like $\mathrm{O}, \mathrm{C}$ and $\mathrm{N}$ are expected to occupy the octahedral sites. Both the sites have trigonal symmetry, the same as that of the host lattice, and hence single interstitials are not expected to cause anelastic effects, unless there is an interaction between the two kinds of interstitials forming an $I-I$ pair (Povolo
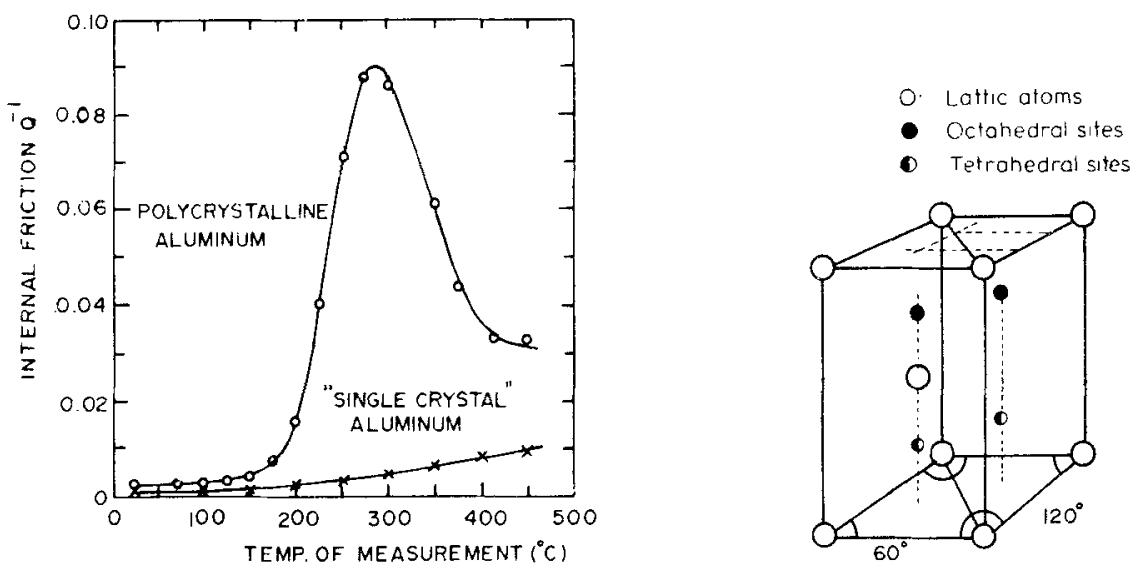

Figure 5. Grain boundary peak in polycrystalline aluminium (Torsional vibration at $0.8 \mathrm{~Hz}$ ).

Figure 6. Interstitial positions in the hexagonal close packed lattice. 


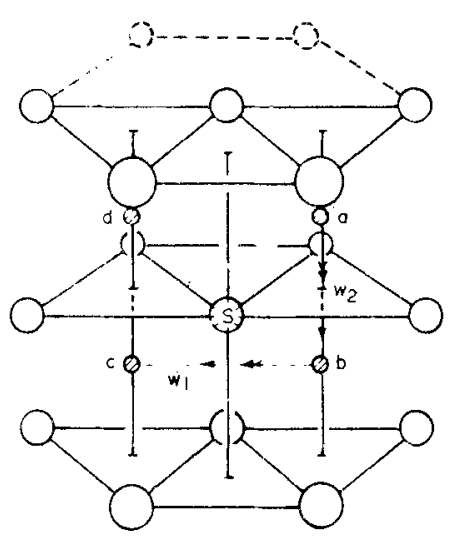

Figure 7. The two types of interstitial jumps producing reorientation of a nearest neighbour $I-S$ pair in the hcp structure. $W_{1}$ is the jump rate parallel to the basal plane and $W_{2}$ is perpendicular to the basal plane.

and Bisogni 1967). This will be possible for small atoms like $H$ which may occupy octahedral as well as tetrahedral sites. In terms of the jump probabilities $W_{1}$ for the transition from tetrahedral to octahedral site and $W_{2}$ for the reverse jump, the relaxation time $\tau$ is given by

$$
\tau^{-1}=W_{1}+W_{2} \text {. }
$$

Relaxation due to interstitial-substitutional $(I-S)$ pair in hexagonal crystals has been discussed by Nowick (1967). The nearest neighbour pairs lie in (100) type planes and the $(I-S)$ defect is of the $\langle 100\rangle$ monoclinic type and two kinds of jumps are possible during the relaxation (figure 7 ). In terms of the probability $W_{1}$ for a jump normal to the basal plane and $W_{2}$ for a jump within the basal plane, the two relaxation times are given by

$$
\begin{aligned}
& \tau_{1}^{-1}=3 W_{2}+2 W_{1}, \\
& \tau_{2}^{-1}=3 W_{2} .
\end{aligned}
$$

In addition to the relaxations due to point defect interactions, relaxaiion effects can also occur due to dislocations and semi-coherent or incoherent interface boundaries.

\subsection{Relaxations associated with interstitials}

5.2a I-I interactions: Relaxation peaks due to hydrogen interstitial pair have been observed by Koster et al (1956) in Ti, by Bungardt and Preisendanz (1960) in Zr and by Hasiguti et al (1965) in zircaloy-2. Miller and Browne (1970) and Browne (1972) reported a relaxation peak due to oxygen interstitial pair in $\mathrm{Ti}, \mathrm{Zr}$ and $\mathrm{Hf}$. For $\mathrm{I}-\mathrm{I}$ interaction the relaxation strength should be proportional to the number of such pairs and hence to the square of the interstitial content. Miller and Browne (1970) reported such behaviour for both Ti-O and Ti-C alloys. However, investigations by others (Misra and Asundi 1972a, b; Povolo and Bisogni 1967) failed to identify the I-I peak in zirconium and titanium. Interstitials were observed to give rise to relaxation only in conjunction with a substitutional atom.

$5.2 \mathrm{~b} \quad I-S$ pair relaxations: Interstitial relaxation in hcp metals involving $I-S$ pairs was first reported by Pratt et al (1954) for Ti containing oxygen. Similar results were reported by Gupta and Weinig (1962) and Bratina (1962). The height of the $I-S$ peak was a linear function of the oxygen content, and was related to the size of the substitutional atom. 
Bisogni et al (1964) measured the relaxation of oxygen and nitrogen in polycrystalline hafnium containing zirconium as substitutional element. Miller (1962) reported an oxygen peak in 'iodide pure' titanium, but the result could be due to the presence of traces of substitutional atoms. Misra and Asundi $(1970,1972)$ made extensive study of the internal friction due to oxygen and nitrogen in $\mathrm{Ti}$ and $\mathrm{Zr}$ with a variety of substitutional atoms having different size factors. In zirconium for all the alloying elements, oxygen gave two peaks, the relaxation strength depending on the concentration of oxygen. At low oxygen concentrations, the high temperature peak was more prominent and was ascribed due to three oxygen atom jumps. At high oxygen concentrations, the low temperature peak was more prominent and was due to a single oxygen jump. In nitrogen, only those substitutional elements with atomic size larger than that of zirconium gave internal friction peaks similar to those for oxygen. In titanium, $S-I$ relaxation peaks were studied for various interstitials like $\mathrm{C}, \mathrm{O}$ and $\mathrm{N}$.

Using the torsion pendulum technique in the frequency range 0.5 to $1.5 \mathrm{~Hz}$ and the temperature range $-190^{\circ} \mathrm{C}$ to $800^{\circ} \mathrm{C}$, Misra (1976) presented extensive results on a series of $\mathrm{Zr}$ and $\mathrm{Ti}$ base dilute alloys.

5.2c I- $V$ pair interactions: In materials with low temperature irradiation damage interstitial-vacancy pairs may cause relaxation effects. Moser et al (1973) have studied I$V$ pair interaction in neutron irradiated bcc, hcp and fcc metals. The influence of oxygen and $\gamma$-irradiation on the elastic modulus and internal friction of iodide pure zirconium has been reported by Federov et al (1971). It has been shown that stable oxygen-vacancy aggregates of various complexities may exist even after annealing. Three relaxation processes associated with oxygen-vacancy complexes are reported in quenched or irradiated zirconium samples.

\subsection{Boundary relaxations}

There are a number of results in the literature pertaining to twin boundary and grain boundary relaxations. Reed-Hill and Dahlberg (1966) studied the elastic after-effect in iodide pure zirconium prestrained at $77^{\circ} \mathrm{K}$. At a prestrain of less than $1 \%$, the microstructure indicated $\{1 \overline{12} 1\}$ twins. It is very likely that the twin boundaries move easily under load. The activation energy obtained in their study $(22.7 \mathrm{kcal} / \mathrm{mol})$ has been interpreted as that for slip ahead of the twin interface.

Studies of internal friction associated with grain boundaries in $\mathrm{Ti}$ and $\mathrm{Zr}$ were reported by Pratt et al (1954), Bratina and Winegard (1956), Bungardt et al (1962) and Misra and Asundi (1972a). Bungardt et al (1962) observed well-developed peaks around $600^{\circ} \mathrm{C}$ at $1 \mathrm{~Hz}$, whose magnitude changes with grain size.

Bedford et al (1972) in their studies on zirconium having different cell structures found an increase in the damping for finer cell structure similar to that observed for grain boundary relaxations for fine grain structure. The high background damping in deformed zirconium is attributed to relaxation of dislocation cell walls. On recovery, dislocation rearrangement results in polygonized sub boundaries and lowering of the damping at higher temperatures.

\subsection{Dislocation relaxations}

The behaviour of dislocations in hexagonal crystals is highly anisotropic because of the limited number of slip systems that can operate in the structure. Consequently, strong 
orientation dependence of dislocation damping effects is to be expected in single crystal experiments.

The Bordoni peak in $\mathrm{Zr}$ and $\mathrm{Ti}$ is generally much smaller than the corresponding peak for fcc metals, probably due to the presence of interstitial impurities. Gruzin and Semenikhin (1963) studied the low temperature damping (Bordoni peak) in high purity zirconium which had been deformed to various amounts using a flextural vibration technique. The effect of alloying as well as fast neutron irradiation on the stability of the peak was studied. Doherty and Gibbons (1971) studied the Bordoni peaks in zirconium using a composite resonator technique in the frequency range of 100 to $150 \mathrm{kHz}$ and found an additional peak towards the low temperature range for a sample cold worked by $38 \%$ reduction in cross-section. Under the application of hydrostatic pressure, both the peaks were observed to increase in height. Fonquet et al (1970) studied $\mathrm{Zr}$ and $\mathrm{Zr}$ base alloys at a frequency of $13 \mathrm{kHz}$.

Hasiguti (1953) found that there exists an internal friction peak above the temperature of the Bordoni peak in cold worked metais. This type of peak known as the Hasiguti peak has been reported in $\mathrm{Ti}, \mathrm{Zr}, \mathrm{Mg}$ (Hasiguti et al 1962) and beryllium (Abrams et al 1966). The Hasiguti peak is usually small and has higher activation energy than the Bordoni peak and is caused by dislocation relaxation. Yukiti and Hasiguti (1972) reviewed the basic mechanism of thermally-activated unpinning of dislocations resulting in the Hasiguti peak and deduced that the peak temperature shifts with stress amplitude.

The most extensive study on single crystals was carried out by Tusi and Sack (1967). Internal friction studies and electron microscopic observations were carried out on $\mathbf{M g}$ single crystals. Figure 8 shows the results for magnesium single crystals after $0.5 \%$ compression. ( $\theta$ is the angle between the crystal axis and the hexagonal axis). A broad peak at around $200^{\circ} \mathrm{K}$ was observed in $2-40 \mathrm{kHz}$ range. From the orientation dependence, it is inferred that dislocation motion in the basal plane is involved in the relaxation process. The peak height increased with deformation and attained a maximum by $2.5 \%$ deformation by compression. The peak height was quite sensitive to the presence of impurities. About $1 \%$ Li completely suppress it, whereas $\mathrm{Fe}$ and $\mathrm{Ni}$ prevent the peak from disappearing even in annealed samples. A small peak at about $20^{\circ} \mathrm{K}$ was noticed in cold worked polycrystals and highly deformed single crystals, presumably due to grain boundary or twin boundary relaxations.

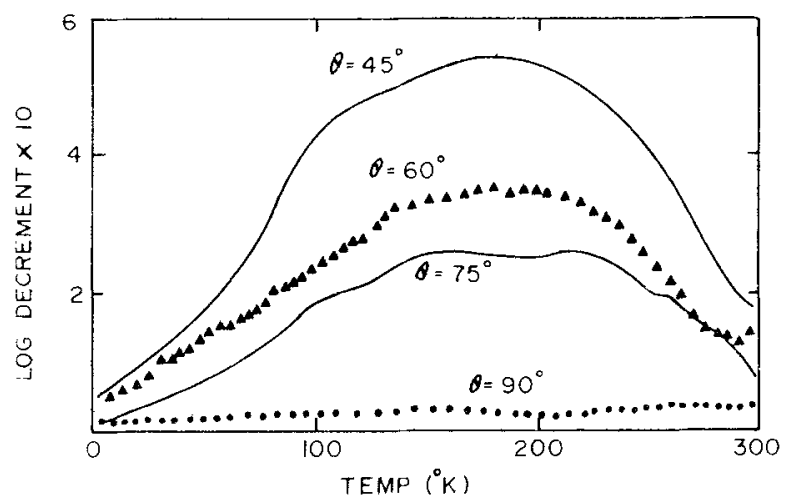

Figure 8 . Internal friction of single crystals of magnesium of different orientations after $0.5 \%$ compression. 
Amplitude-dependent internal friction in $\mathrm{Mg}$ single crystals has been studied by Roberts (1968) in the temperature range of $80-320^{\circ} \mathrm{K}$ at frequencies 0.015 to $0.75 \mathrm{~Hz}$. Very broad peaks were observed indicating the possibility of a large number of relaxation processes occurring together. The results are interpreted in terms of thermally activated dislocation-dislocation interactions. (Roberts and Hartman 1963; Alefeld 1962).

Esnouf and Fantozzi (1981) observed a strong internal friction peak at about half the melting temperature in high purity polycrystalline $\mathrm{Mg}$. The position of the peak was dependent on strain amplitude. The results are explained in terms of dislocation glide controlled by jog climbing and diffusion of vacancies along dislocations. Atrens (1974) studied the interaction of oxygen as pinning points for dislocations in zirconium. In deformed and annealed zirconium, Ritchie and Sprungmann (1981) observed three internal friction peaks in the temperature range of 480 to $600^{\circ} \mathrm{C}$ and interpreted them in terms of dislocation core diffusion and unpinning of dislocations.

\section{Conclusion}

Compared to bcc and fcc metals, the experimental studies on hexagonal metals are fewer and various aspects are to be investigated in detail for a better understanding of the relaxation processes in hexagonal metals. Many of the results were obtained on polycrystalline materials in the form of wires or rods and hence subject to pronounced texture effects. Single crystal studies are desirable for elucidating the orientation effects on dislocation interactions. Such results would be of considerable value in understanding the point defect and dislocation interactions in hep metals.

\section{References}

Abrams E H, Kamber K T and Ang C Y 1966 Acta. Metall. 14729

Alefeld G 1962 Z. Phys. 170249

Atrens A 1974 Scr. Metall. 8401

Bedford A J, Fuller P G and Miller D R 1972 J. Nucl. Mater. 43164

Berry B S 1962 Acta Metall. 10271

Bisogni E, Mat G and Wert C 1964 J. Less Common Metals 7197

Brailsford A D 1961 Phys. Rev. 122778

Bratina W J 1962 Acta Metall. 10332

Bratina W J and Winegard W C 1956 Trans. AIME 206186

Bommel H E 1954 Phys. Rev. 96220

Browne K M 1972 Acta Metall. 20507

Bungardt K and Preisendanz H 1960 Z. Metall. 51280

Bungardt, Preisendanz H and Horn E 1956 Z. Metall. 53495

Burdett C F and Queen T J 1970 Metall. Rev. 14347

Cochardt A 1959 in Magnetic properties of metals and alloys (ed) R M Bozorth, (Cleveland, Ohio: Am., Soc. Metals) 251

De Batist R 1972 Internal friction of structural defects in crystalline solids (Amsterdam: North-Holland Publishing Company)

De Fouquet J, Boch P, Petit J and Hisu G 1970 J. Phys. Chem. Solids 311901

Doherty J E and Gibbons D F 1971 J. Appl. Phys. 42

Entwistle K M 1962 Metall. Rev. 7175

Esnouf C and Fantozzi G 1981 J. Phys. Colloq (France) 42 C-5 445

Fantozzi G and Ritchie I G 1981 J. Phys. Colloq. (France) 42 C-5 3

Fedorov G B, Kissil A Ye and Festisov G V 1971 Fiz. Met. Metalloved 32621 
Granato A V and Lucke K 1956 J. Appl. Phys. 27 583, 789

Gruzin P L and Semenikhin A N 1963 Fiz. Met. Metalloved 15791

Gupta D and Weinig S 1962 Acta Metall. 10292

Hasiguti R R 1953 Proc. Int. Conf. Theor. Phys., Kyoto and Tokyo 577

Hasiguti R R, Igata N and Kamoshita G 1962 Acta Metall. 10442

Hasiguti R R, Igata N, Tonomae R, Nakumara Y, Saso S and Domato K 1965 Symp. Nondestructive tesing in nuclear technology, Bucharest

Hoffman R A and Wert C 1966 J. Appl. Phys. 37237

Ke T S 1949 J. Appl. Phys. 20274

Koster W, Bangert L and Evers M 1956 Z. Metall. 47567

Lucke K and Granato A V 1957 in Dislocations and mechanical properties of metals (ed) J Fischer, (New York: Wiley)

Mason W P (ed) 1966 Physical acoustics (New York: Academic Press) Vol III

Miller D 1962 Trans. Met. Soc. AIME 224275

Miller D and Browne K M 1970The science technology and applications of titanium (London: Pergamon) 401

Misra S 1976 Anelastic and internal friction behaviour of zirconium and titanium and their alloys Ph.D Thesis, The Banaras Hindu University.

Misra S and Asundi M K 1970 Trans. Indian Inst. Metals 2373 1972a Can. Met. Q. 1169

Misra S and Asundi M K 1972b Titanium science and technology (ed) R I Jaffe and H M Burte (New York: Plenum Press)

Moser P, Verdone J, Chambron W, Nivert V and Pichon R 1973 5th Int. Conf. Internal Friction and Ultrasonic Attenuation in Crystalline Solids, Achen

Niblet D H and Wilks J 1956 Philos Mag 1415

Niblet D H and Wilks J 1960 Adv. Phys. 91

Nowick A S 1953 Prog. Metal Phys. 41

Nowick A S 1967 Adv. Phys. 161

Nowick A S and Berry B S 1972 Anelastic relaxation in crystalline solids (New York: Academic Press)

Povolo F and Bisogni E A 1967 Acta Metall. 15701

Pratt J N, Bratina W J and Chalmers B 1954 Acta Metall. 2203

Read T A 1940 Phys. Rev. 58371

Reed-Hill R E and Dahlberg K P 1966 J. Elec. Chem. Technol 4303

Ritchie I G and Sprungmann K W 1981 J. Phys. Colloq (France) 42 C-5 427

Roberts J M 1968 Trans. Jpn Inst. Metals 969

Roberts J M and Hartmann D E 1963 J. Phys. Soc. Jpn 18119

Snoek J L 1941 Physica 8711

Snoek J L 1942 Physica 9862

Tusi R T C and Sack H S 1967 Acta Metall. 151715

Waterman P C 1958 J. Appl. Phys. 291190

Wilks J 1965 Brit. J. Appl. Phys. 16587

Yukuti R and Hasiguti R R 1972 J. Less Common Metals 28249

Zener C 1943 Trans. AIME 152122

Zener C 1947 Phys. Rev. 7134

Zener C 1948 Elasticity and anelasticity of metals (Chicago: University Press) 